PROCEEDINGS OF THE

AMERICAN MATHEMATICAL SOCIETY

Volume 132, Number 9, Pages 2799-2807

S 0002-9939(04)07413-1

Article electronically published on April 21, 2004

\title{
ON SIMULTANEOUS LINEAR EXTENSIONS OF PARTIAL (PSEUDO)METRICS
}

\author{
E. D. TYMCHATYN AND M. ZARICHNYI
}

(Communicated by Alan Dow)

\begin{abstract}
We consider the question of simultaneous extension of (pseudo) metrics defined on nonempty closed subsets of a compact metrizable space. The main result is a counterpart of the result due to Künzi and Shapiro for the case of extension operators of partial continuous functions and includes, as a special case, Banakh's theorem on linear regular operators extending (pseudo)metrics.
\end{abstract}

\section{INTRODUCTION}

The theories of continuous extensions of continuous functions and continuous metrics develop in parallel. Hausdorff's theorem on extension of a metric defined on a closed subset of a metrizable space [12] is a counterpart of the Tietze-Urysohn theorem on extensions of continuous functions. Hausdorff's theorem has had many improvements; see, for example, 6, 19, 22].

The set of continuous pseudometrics on a compact metrizable $X$ forms a positive cone in the normed space $C(X \times X)$ of continuous functions on $X \times X$. C. Bessaga in [4, 5] formulated the problem of existence of continuous linear operators that extend (pseudo)metrics defined on a closed subset of $X$. These operators resemble the linear extension operators for continuous functions (see [7]). In some special cases, Bessaga solved this problem; his solution is based on an explicit formula for extension of metrics onto the so-called squeezed cone over a space. In its general setting, the extension problem for pseudometrics was first solved by T. Banakh [1] by improving Bessaga's formulas, and the proof was completed by O. Pikhurko [20]. In [2, T. Banakh also developed another approach to the extension problem. A very short proof of the existence of the linear operators extending (pseudo)metrics is given in 23 . Note that some sublinear extension operators for (pseudo)metrics were constructed in [18].

Recently, Künzi and Shapiro [17] considered a problem of simultaneous extension of partial continuous functions. The spaces of partial functions (i.e., the functions defined on various subsets of topological spaces), first defined in 14, 15, naturally appear in the topological theory of differential equations (see 9]). In the present paper, we consider the analogous problem for partial (pseudo)metrics, i.e.,

Received by the editors November 18, 2002 and, in revised form, May 29, 2003.

2000 Mathematics Subject Classification. Primary 54E35, 54C20, 54E40.

The paper was finished during the second named author's visit to the University of Saskatchewan. This research was supported in part by NSERC research grant OGP 005616. 
(pseudo)metrics defined on closed subsets of a compact metrizable space. Note that the metric spaces with variable domains are of interest in different areas of geometry and topology. In particular, the (classes of equivalence up to isometry of) compact metric spaces are the points of the Hausdorff moduli space (see, e.g., [11).

The main result of the paper is a theorem whose formulation is a mixture of those of the cited theorems proved by Banakh and by Künzi and Shapiro: There exists a continuous operator that extends (pseudo)metrics defined on all closed subsets of a compact metrizable space over all the space and whose restriction on the set of pseudometrics defined on every single subset is linear and regular (i.e., of norm 1; see the definitions below).

The authors express their gratitude to an anonymous referee for correcting several misprints and improving the text of the paper.

\section{Preliminaries}

Let $X$ be a metrizable separable space. To avoid trivialities we always assume that $|X| \geq 2$. Let $\Delta_{X}$ denote the diagonal of $X$. Let $I=[0,1]$.

By $\exp X$ we denote the hyperspace of $X$, i.e., the set of all nonempty compact subsets of $X$ endowed with the Vietoris topology. A base of this topology consists of the sets of the form

$$
\left\langle U_{1}, \ldots, U_{k}\right\rangle=\left\{A \in \exp X \mid A \subset \bigcup_{i=1}^{k} U_{i}, A \cap U_{i} \neq \emptyset \text { for all } i\right\}
$$

where $U_{1}, \ldots, U_{k}$ run over the family of open subsets in $X$.

If $d$ is a compatible metric on $X$, then the Vietoris topology is generated by the Hausdorff metric $d_{H}$,

$$
d_{H}(A, B)=\inf \left\{\varepsilon>0 \mid A \subset O_{\varepsilon}(B), B \subset O_{\varepsilon}(A)\right\} .
$$

Given a nonempty compact subset $A$ of $X$, we denote by $\mathcal{M}(A)$ (respectively $\mathcal{P} \mathcal{M}(A))$ the set of continuous metrics (respectively pseudometrics) on $A$ (the fact that $\varrho \in \mathcal{P} \mathcal{M}(A)$ will also be expressed as $\operatorname{dom} \varrho=A)$. Set $\mathcal{M}=\bigcup\{\mathcal{M}(A) \mid A \in$ $\exp X\}, \mathcal{P} \mathcal{M}=\bigcup\{\mathcal{P} \mathcal{M}(A) \mid A \in \exp X\}$.

Identifying every pseudometric $d \in \mathcal{P} \mathcal{M}$ with its graph, which is a compact subset of $X \times X \times \mathbb{R}$, we consider the set $\mathcal{P} \mathcal{M}$ as a subset of $\exp (X \times X \times \mathbb{R})$ and endow $\mathcal{P} \mathcal{M}$ with the subspace topology.

Let $K=\{(\varrho, \lambda) \in \mathcal{P} \mathcal{M} \times \mathcal{P} \mathcal{M} \mid \operatorname{dom} \varrho=\operatorname{dom} \lambda\}$. The space $\mathcal{P} \mathcal{M}$ is a positive cone in the following sense: the maps $(c, \varrho) \mapsto c \varrho$ and $(\varrho, \lambda) \mapsto \varrho+\lambda$ are continuous as maps from, respectively, $\mathbb{R}_{+} \times \mathcal{P} \mathcal{M}$ and $K$ into $\mathcal{P} \mathcal{M}$.

A map $u: \mathcal{P} \mathcal{M} \rightarrow \mathcal{P} \mathcal{M}(X)$ is called linear if (i) $u(c \varrho)=c u(\varrho)$ for every $c \in \mathbb{R}_{+}$ and $\varrho \in \mathcal{P} \mathcal{M}$, and (ii) $u(\varrho+\lambda)=u(\varrho)+u(\lambda)$ for every $(\varrho, \lambda) \in K$.

The norm of $\varrho \in \mathcal{P} \mathcal{M}$ is $\|\varrho\|=\sup \{\varrho(x, y) \mid x, y \in \operatorname{dom} \varrho\}$.

A map $u: \mathcal{P} \mathcal{M} \rightarrow \mathcal{P} \mathcal{M}(X)$ is called regular if $\|u(\varrho)\|=\|\varrho\|$ for every $\varrho \in \mathcal{P} \mathcal{M}$.

A map $u: \mathcal{P} \mathcal{M} \rightarrow \mathcal{P} \mathcal{M}(X)$ is called an extension operator if

$$
u(\varrho) \mid(\operatorname{dom} \varrho \times \operatorname{dom} \varrho)=\varrho, \text { for every } \varrho \in \mathcal{P} \mathcal{M}
$$




\section{A SELECTION THEOREM FOR MULTI-VALUED MAPPINGS}

Let $\langle T, \mathcal{A}, \mu\rangle$ be a measure space. For a Banach space $B$ with norm $\|\cdot\|$, we denote by $L_{1}(T, B)$ the Banach space of functions from $T$ to $B$ integrable in the Bochner sense. The norm in $L_{1}(T, B)$ is defined by the formula $\|\alpha\|=\int_{T}\|\alpha(t)\| d \mu$.

A set $Z$ of measurable mappings from $\langle T, \mathcal{A}, \mu\rangle$ into a topological space $X$ is said to be decomposable (see, e.g., [13], [16]) if for every $f, g \in Z$ and every $A \in \mathcal{A}$ the mapping

belongs to $Z$.

$$
h(t)= \begin{cases}f(t), & t \in A, \\ g(t), & t \notin A,\end{cases}
$$

Note that every set in $L_{1}(T, B)$ of cardinality 1 is decomposable. Besides, for every closed subset $A$ of $B$ the set $L_{1}(T, A)=\left\{\alpha \in L_{1}(T, B) \mid \alpha(T) \subset A\right\}$ is closed decomposable.

Recall that a multi-valued mapping $F: X \rightarrow Y$ is called lower semicontinuous provided that $\{x \in X \mid F(x) \cap U \neq \emptyset\}$ is open in $X$ whenever $U$ is open in $Y$.

We will need the following special case of a selection theorem due to Fryszkowski [10] for the unit interval $I=[0,1]$ with the Lebesgue measure $m$.

Theorem 3.1. Let $B$ be a separable Banach space. Every lower semicontinuous mapping $F$ from a metric compactum $X$ into $L_{1}(I, B)$ with closed decomposable values admits a continuous single-valued selection.

\section{Construction}

Assume that a compact metric space $X$ is embedded into a Banach space $(B,\|\cdot\|)$. Define a multi-valued map $F: \exp X \times X \rightarrow L_{1}(I, B)$ as follows:

$$
F(A, x)= \begin{cases}\{x\}=L_{1}(I,\{x\}), & \text { if } x \in A, \\ L_{1}(I, A), & \text { if } x \notin A .\end{cases}
$$

Proposition 4.1. The map $F$ is lower semicontinuous.

Proof. We have to show that for every open subset $U$ of $L_{1}(I, B)$ the set

$$
U^{\sharp}=\{(A, x) \in \exp X \times X \mid F(A, x) \cap U \neq \emptyset\}
$$

is open in $\exp X \times X$. Let $(A, x) \in U^{\sharp}$. Consider two cases.

Case 1). $x \notin A$. Then $F(A, x)=L_{1}(I, A)$. There exist $\alpha \in L_{1}(X, A) \cap U$ and $\varepsilon>0$ such that $O_{\varepsilon}(\alpha) \subset U$. There exists a neighborhood $V$ of $(A, x)$ in $\exp X \times X$ such that $d_{H}\left(A, A^{\prime}\right)<\varepsilon / 2$ and $x^{\prime} \notin A^{\prime}$ for every $\left(A^{\prime}, x^{\prime}\right) \in V$.

There exists $\beta \in L_{1}(I, A)$ such that $\|\alpha-\beta\|<\varepsilon / 2$ and $\beta$ takes a finite number of values (see, e.g., [8]). One can perturb the values of $\beta$ to obtain a function $\beta^{\prime} \in L_{1}\left(I, A^{\prime}\right)$ with the property that $\left\|\beta(t)-\beta^{\prime}(t)\right\|<\varepsilon / 2$ for every $t \in I$.

Since $d\left(\alpha(t), \beta^{\prime}(t)\right)<\varepsilon$ for all $t$, we have $\left\|\alpha-\beta^{\prime}\right\|<\varepsilon$, and therefore $\beta^{\prime} \in U$. Hence, $V \subset U^{\sharp}$.

Case 2). $x \in A$. Then $F(A, x)=\{x\}$ (as above, we identify $x \in X$ with the constant function taking $x$ as its value). Since $x \in U$ and $U$ is open, there exists $\varepsilon>0$ such that $O_{\varepsilon}(x) \subset U$. Then there is a neighborhood $V$ of $(A, x)$ in $\exp X \times X$ such that $d_{H}\left(A, A^{\prime}\right)<\varepsilon$ and $d\left(x, x^{\prime}\right)<\varepsilon$ for every $\left(A^{\prime}, x^{\prime}\right) \in V$. For $\left(A^{\prime}, x^{\prime}\right) \in V$, denote by $\alpha^{\prime}$ the constant map whose value is $x^{\prime}$ whenever $x^{\prime} \in A^{\prime}$ and arbitrary $y \in A^{\prime}$ such that $d(x, y)<\varepsilon$ whenever $x^{\prime} \notin A^{\prime}$. Then $\alpha^{\prime} \in F\left(A^{\prime}, x^{\prime}\right) \cap U$. 
Since $F$ satisfies the hypotheses of Theorem [3.1, there exists a continuous selection $f: \exp X \times X \rightarrow L_{1}(I, B)$ of $F$. Define $u=u_{f}: \mathcal{P} \mathcal{M} \rightarrow \mathcal{P} \mathcal{M}(X)$ by the formula

$$
u(\varrho)(x, y)=\int_{0}^{1} \varrho(f(\operatorname{dom}(\varrho), x)(t), f(\operatorname{dom}(\varrho), y)(t)) d t .
$$

Lemma 4.2. Let $\varrho \in \mathcal{M}(X)$. For every $\varepsilon>0$ there exists $\delta>0$ such that $\int_{0}^{1} \varrho(\alpha(t), \beta(t)) d t<\varepsilon$ whenever $\alpha, \beta \in L_{1}(I, B)$ with $\|\alpha-\beta\|<\delta$.

Proof. We may assume that $|X| \geq 2$. Since $\varrho$ is uniformly continuous on $X \times X$, there exists $\delta_{1}>0$ such that $\varrho(x, y)<\varepsilon / 2$ whenever $\|x-y\|<\delta_{1}$. Let $0<\delta<$ $\min \left\{\delta_{1}, \frac{\varepsilon \delta_{1}}{2 \operatorname{diam} X}\right\}$ (here diam $X$ is the diameter of $X$ with respect to the metric on $X$ induced by the norm in $B)$. Then for every $\alpha, \beta \in L_{1}(I, X)$ with $\|\alpha-\beta\|<\delta$ we have $m(A)<\frac{\varepsilon}{2 \operatorname{diam} X}$, where $A=\left\{t \mid\|\alpha(t)-\beta(t)\| \geq \delta_{1}\right\}$ ) (recall that $m$ denotes the Lebesgue measure on $I)$. Therefore,

$$
\begin{aligned}
& \int_{0}^{1} \varrho(\alpha(t), \beta(t)) d t=\int_{A} \varrho(\alpha(t), \beta(t)) d t+\int_{I \backslash A} \varrho(\alpha(t), \beta(t)) d t \\
< & \frac{\varepsilon}{2 \operatorname{diam} X} \operatorname{diam} X+\int_{I \backslash A} \frac{\varepsilon}{2} d t \leq \varepsilon .
\end{aligned}
$$

Lemma 4.3. Let $\varrho \in \mathcal{P} \mathcal{M}(X)$. The map $q=q_{\varrho}: L_{1}(I, X) \times L_{1}(I, X) \rightarrow \mathbb{R}$ defined by $q(\alpha, \beta)=\int_{0}^{1} \varrho(\alpha(t), \beta(t)) d t$ is uniformly continuous.

Proof. Let $\varepsilon>0$. By Lemma 4.2 one can find $\delta>0$ such that for every $\alpha, \beta \in$ $L_{1}(I, X)$ we have $\int_{0}^{1} \varrho(\alpha(t), \beta(t)) d t<\frac{\varepsilon}{2}$ whenever $\|\alpha-\beta\|<\delta$. Now let $\alpha_{1}, \beta_{1}, \alpha_{2}, \beta_{2}$ $\in L_{1}(I, X)$ be such that $\left\|\alpha_{1}-\alpha_{2}\right\|<\delta,\left\|\beta_{1}-\beta_{2}\right\|<\delta$. Then

$$
\begin{aligned}
& \quad\left|q\left(\alpha_{1}, \beta_{1}\right)-q\left(\alpha_{2}, \beta_{2}\right)\right|=\left|\int_{0}^{1}\left(\varrho\left(\alpha_{1}(t), \beta_{1}(t)\right)-\varrho\left(\alpha_{2}(t), \beta_{2}(t)\right)\right) d t\right| \\
& \leq\left|\int_{0}^{1}\left(\varrho\left(\alpha_{1}(t), \beta_{1}(t)\right)-\varrho\left(\alpha_{1}(t), \beta_{2}(t)\right)\right) d t\right| \\
& \quad \quad+\left|\int_{0}^{1}\left(\varrho\left(\alpha_{1}(t), \beta_{2}(t)\right)-\varrho\left(\alpha_{2}(t), \beta_{2}(t)\right)\right) d t\right| \\
& \leq \int_{0}^{1} \rho\left(\beta_{1}(t), \beta_{2}(t)\right) d t+\int_{0}^{1} \rho\left(\alpha_{1}(t), \alpha_{2}(t)\right) d t \\
& \leq \frac{\varepsilon}{2}+\frac{\varepsilon}{2}=\varepsilon .
\end{aligned}
$$

Proposition 4.4. Let $\varrho \in \mathcal{P} \mathcal{M}$. The function $u(\varrho)$ is a continuous pseudometric on $X$.

Proof. It is evident that $u(\varrho)$ is a pseudometric on $X$.

Let $\left(x_{i}\right),\left(y_{i}\right)$ be convergent sequences in $X$ with the limits $x$ and $y$ respectively. Given $\varepsilon>0$, by Lemma 4.3 one can find $\delta>0$ such that $\left|q_{\varrho}\left(\alpha_{1}, \beta_{1}\right)-q_{\varrho}\left(\alpha_{2}, \beta_{2}\right)\right|<\varepsilon$ 
whenever $\left\|\alpha_{1}-\alpha_{2}\right\|<\delta$ and $\left\|\beta_{1}-\beta_{2}\right\|<\delta$ for $\alpha_{1}, \alpha_{2}, \beta_{1}, \beta_{2} \in L_{1}(I$, dom $\varrho)$. There exists $N \in \mathbb{N}$ such that for every $n>N$,

$$
\begin{aligned}
& \left\|f\left(\operatorname{dom} \varrho, x_{n}\right)-f(\operatorname{dom} \varrho, x)\right\|<\delta, \\
& \left\|f\left(\operatorname{dom} \varrho, y_{n}\right)-f(\operatorname{dom} \varrho, y)\right\|<\delta .
\end{aligned}
$$

Then $\left|u(\varrho)\left(x_{n}, y_{n}\right)-u(\varrho)(x, y)\right|<\varepsilon$ for every $n>N$.

Proposition 4.5. The map $u$ is continuous.

Proof. Let $\left(\varrho_{n}\right)$ be a sequence in $\mathcal{P} \mathcal{M}$ and $\varrho_{n} \rightarrow \varrho \in \mathcal{P M}$. Then, obviously, dom $\varrho_{n} \rightarrow$ dom $\varrho$. Denote by $\varrho$ an extension of $\varrho$ over $X$. Denote by $q: L_{1}(I, X) \times$ $L_{1}(I, X) \rightarrow \mathbb{R}$ the map defined by the formula

$$
q(\alpha, \beta)=\int_{0}^{1} \tilde{\varrho}(\alpha(t), \beta(t)) d t, \quad \alpha, \beta \in L_{1}(I, X) .
$$

As we have shown in Lemma 4.3, $q$ is a uniformly continuous pseudometric on $L_{1}(I, X)$.

Let $\tilde{\varrho}_{n}=\tilde{\varrho} \mid\left(\operatorname{dom} \varrho_{n} \times \operatorname{dom} \varrho_{n}\right)$. Obviously, $\tilde{\varrho}_{n} \rightarrow \varrho$ in $\mathcal{P} \mathcal{M}$ (see, e.g., 17]).

Let $\varepsilon>0$. We are going to show that there exists $n \in \mathbb{N}$ such that for every $n \in \mathbb{N}$ with $n>N$ we have $\left.\mid u(\varrho)(x, y)-u\left(\tilde{\varrho}_{n}\right)(x, y)\right) \mid<\varepsilon$.

Since $q$ is uniformly continuous, there exists $\delta>0$ such that for every $\alpha, \beta \in$ $L_{1}(I, X)$ with $\|\alpha-\beta\|<\delta$ we have $q(\alpha, \beta)<\frac{\varepsilon}{2}$.

There exists $N_{1} \in \mathbb{N}$ such that for every $n \in \mathbb{N}$ with $n>N_{1}$ we have

$$
\max \left\{\left|\tilde{\varrho}(x, y)-\varrho_{n}(x, y)\right| \mid(x, y) \in \operatorname{dom} \varrho_{n}\right\}<\frac{\varepsilon}{2} .
$$

Moreover, there exists $N_{2} \in \mathbb{N}$ such that for every $n \in \mathbb{N}$ with $n>N_{2}$ and for every $x \in X$ we have $\left\|f(\operatorname{dom} \varrho, x)-f\left(\operatorname{dom} \varrho_{n}, x\right)\right\|<\delta$.

Let $N=\max \left\{N_{1}, N_{2}\right\}, n>N$, and $x, y \in X$. Then

$$
\begin{aligned}
& \left|u(\varrho)(x, y)-u\left(\varrho_{n}\right)(x, y)\right| \\
& =\mid \int_{0}^{1} \varrho(f(\operatorname{dom} \varrho, x)(t), f(\operatorname{dom} \varrho, y)(t)) d t \\
& -\int_{0}^{1} \varrho_{n}\left(f\left(\operatorname{dom} \varrho_{n}, x\right)(t), f\left(\operatorname{dom} \varrho_{n}, y\right)(t)\right) d t \mid \\
& \leq \mid \int_{0}^{1}(\varrho(f(\operatorname{dom} \varrho, x)(t), f(\operatorname{dom} \varrho, y)(t)) \\
& \text { - } \varrho\left(f\left(\operatorname{dom} \varrho_{n}, x\right)(t), f\left(\operatorname{dom} \varrho_{n}, y\right)(t)\right) d t \\
& +\mid \int_{0}^{1}\left(\tilde{\varrho}\left(f\left(\operatorname{dom} \varrho_{n}, x\right)(t), f\left(\operatorname{dom} \varrho_{n}, y\right)(t)\right)\right. \\
& -\varrho_{n}\left(f\left(\operatorname{dom} \varrho_{n}, x\right)(t), f\left(\operatorname{dom} \varrho_{n}, y\right)(t)\right) d t \\
& \leq \frac{\varepsilon}{2}+\frac{\varepsilon}{2}=\varepsilon \text {. }
\end{aligned}
$$


The main result of this section is the following theorem, which we improve in the next section.

Theorem 4.6. There exists a continuous regular linear extension operator $u: \mathcal{P} \mathcal{M}$ $\rightarrow \mathcal{P M}(X)$.

Proof. It follows from Propositions 4.4 and 4.5 that $u: \mathcal{P M} \rightarrow \mathcal{P M}(X)$ is welldefined and continuous. Obviously, $u$ is an extension operator. Its regularity and linearity are easy consequences of the definition.

\section{Operators PRESERVING METRICS}

The operator $u$ constructed in the previous section, in general, does not preserve metrics, i.e., in general, $u(\mathcal{M}) \not \subset \mathcal{M}(X)$. Note that there is no linear operator $\tilde{u}: \mathcal{P} \mathcal{M} \rightarrow \mathcal{P} \mathcal{M}(X)$ such that $\tilde{u}(\mathcal{M}) \subset \mathcal{M}(X)$. Indeed, for every $a \in X$ we have $\mathcal{P} \mathcal{M}(\{a\})=\mathcal{M}(\{a\})=\{0\}$, and it easily follows from the linearity that $\tilde{u}(0)=0$.

Let

$$
\tilde{\mathcal{M}}=\bigcup\{\mathcal{M}(A)|A \in \exp X,| A \mid \geq 2\}
$$

Theorem 5.1. There exists a regular extension operator $\tilde{u}: \mathcal{P} \mathcal{M} \rightarrow \mathcal{P} \mathcal{M}(X)$ such that $\tilde{u}(\tilde{\mathcal{M}}) \subset \mathcal{M}(X)$.

Proof. We are going to modify $u$ from Theorem 4.6 as follows.

Given $(x, y) \in X \times X \backslash \Delta_{X}$ (as usual, $\Delta_{X}$ denotes the diagonal in $\left.X \times X\right)$ and $A \in$ $\exp X$ with $|A| \geq 2$, we define a multi-valued map $F_{(x, y, A)}: \exp X \times X \rightarrow L_{1}(I, X)$ as follows. Choose points $a_{x}, a_{y} \in A$ so that $a_{x} \neq a_{y}$ and $a_{x}=x$ (respectively $a_{y}=y$ ) if $x \in A$ (respectively $y \in A$ ). Then

$$
F_{(x, y, A)}(B, z)= \begin{cases}F(B, z) & \text { whenever }(A, x) \neq(B, z) \neq(A, y), \\ \left\{a_{x}\right\} & \text { whenever }(B, z)=(A, x), \\ \left\{a_{y}\right\} & \text { whenever }(B, z)=(A, y)\end{cases}
$$

(here $F$ is the multi-valued map from Section 4).

Note that the multi-valued map $F_{(x, y, A)}$ is lower semicontinuous. Indeed, let $U$ be an open subset of $L_{1}(I, X)$. Since $F$ is lower semicontinuous, the set

$$
U^{\sharp}=\left\{\left(A^{\prime}, x^{\prime}\right) \in \exp X \times X \mid F\left(A^{\prime}, x^{\prime}\right) \cap U \neq \emptyset\right\}
$$

is open in $\exp X \times X$. Then the set

$$
\begin{aligned}
& \left\{\left(A^{\prime}, x^{\prime}\right) \in \exp X \times X \mid F_{(x, y, A)}\left(A^{\prime}, x^{\prime}\right) \cap U \neq \emptyset\right\} \\
= & U^{\sharp} \backslash\left\{(A, z) \mid z \in\{x, y\},\left\{a_{z}\right\} \notin U\right\}
\end{aligned}
$$

is open in $\exp X \times X$.

Obviously, the images of $F_{(x, y, A)}$ are closed and decomposable. By the Fryszkowski theorem, there exists a continuous selection $f_{(x, y, A)}$ of $F_{(x, y, A)}$. Since

$$
f_{(x, y, A)}(A, x) \neq f_{(x, y, A)}(A, y),
$$

there exist neighborhoods $W_{A}$ of $A$ in $\exp X$ and $V_{(x, y)}$ of $(x, y)$ in $X \times X$ respectively such that for every $A^{\prime} \in W_{A},\left(x^{\prime}, y^{\prime}\right) \in V_{(x, y)}$ we have

$$
f_{(x, y, A)}\left(A^{\prime}, x^{\prime}\right) \neq f_{(x, y, A)}\left(A^{\prime}, y^{\prime}\right) .
$$


Let

$$
Z=\left\{(x, y, A)|A \in \exp X,| A \mid \geq 2,(x, y) \in(X \times X) \backslash \Delta_{X}\right\} .
$$

The cover $\mathcal{W}=\left\{V_{(x, y)} \times W_{A} \mid(x, y, A) \in Z\right\}$ of $Z$ contains a countable subcover $\mathcal{W}^{\prime}=\left\{V_{\left(x_{i}, y_{i}\right)} \times W_{A_{i}} \mid i \in \mathbb{N}\right\}$ of $Z$.

For $i \in \mathbb{N}$, let $u_{i}=u_{f_{\left(x_{i}, y_{i}, A_{i}\right)}}$. Let $\tilde{u}=\sum_{i \in \mathbb{N}} \frac{u_{i}}{2^{2}}$. Obviously, $\tilde{u}$ is a well-defined map from $\mathcal{P} \mathcal{M}$ to $\mathcal{P} \mathcal{M}(X)$. We leave to the reader a simple verification of the fact that $\tilde{u}$ is a regular extension operator of partial pseudometrics on $X$.

We are going to show that $\tilde{u}(\tilde{\mathcal{M}}) \subset \mathcal{M}(X)$. Let $\varrho \in \tilde{\mathcal{M}}$ and $x, y \in X, x \neq y$. There exists $i \in \mathbb{N}$ such that $(x, y$, dom $\varrho) \in V_{\left(x_{i}, y_{i}\right)} \times W_{A_{i}}$. Then

$$
u_{i}(\varrho)(x, y)=\int_{0}^{1} \varrho\left(f_{\left(x_{i}, y_{i}, A_{i}\right)}(\operatorname{dom} \varrho, x)(t), f_{\left(x_{i}, y_{i}, A_{i}\right)}(\operatorname{dom} \varrho, y)(t)\right) d t>0,
$$

and therefore $\tilde{u}(\varrho)(x, y)>0$. This proves that $\tilde{u}(\varrho)$ is a metric on $X$.

\section{NON-METRIZABLE CASE}

In the case of a non-metrizable compact Hausdorff space $X$, there are no continuous operators of extension of partial continuous functions (E. Stepanova [21]).

One can ask whether there exists an extension operator $u: \mathcal{P} \mathcal{M} \rightarrow \mathcal{P} \mathcal{M}(X)$, for a non-metrizable compact Hausdorff space $X$.

Theorem 6.1. For a compact Hausdorff space $X$ the following conditions are equivalent:

(1) there exists a continuous extension operator $u: \mathcal{P} \mathcal{M} \rightarrow \mathcal{P} \mathcal{M}(X)$;

(2) there exists a continuous map $\Psi:(X \times X) \backslash \Delta_{X} \rightarrow \mathcal{P} \mathcal{M}(X),(x, y) \mapsto \Psi_{(x, y)}$, with $\Psi_{(x, y)}(x, y) \neq 0$ for all $(x, y) \in X^{2} \backslash \Delta_{X}$;

(3) $X$ is metrizable.

Proof. (1) $\Rightarrow(2)$. Let $u: \mathcal{P M} \rightarrow \mathcal{P} \mathcal{M}(X)$ be a continuous extension operator. Denote by $(x, y) \mapsto v_{(x, y)}: X^{2} \backslash \Delta_{X} \rightarrow \mathcal{P} \mathcal{M}$ the map defined by the conditions dom $v_{(x, y)}=\{x, y\}, v_{(x, y)}(x, y)=1$. Then the map $\Psi: X^{2} \backslash \Delta_{X} \rightarrow \mathcal{P} \mathcal{M}(X)$, $(x, y) \mapsto \Psi_{(x, y)}=u\left(v_{(x, y)}\right)$ has the property that $\Psi_{(x, y)}(x, y)=1$.

$(2) \Rightarrow(3)$. Given a continuous map $\Psi:(X \times X) \backslash \Delta_{X} \rightarrow \mathcal{P} \mathcal{M}(X),(x, y) \mapsto \Psi_{(x, y)}$, with $\Psi_{(x, y)}(x, y) \neq 0$ for all $(x, y) \in X^{2} \backslash \Delta_{X}$, define $\Phi:(X \times X) \backslash \Delta_{X} \rightarrow C(X)$ by the formula $\Phi_{(x, y)}(z)=\Psi_{x, y}(x, z), z \in X$. Then $\Phi_{(x, y)}(x)=0 \neq \Phi_{(x, y)}(y)$ for all $(x, y) \in X^{2} \backslash \Delta_{X}$. Therefore, the map $\Phi$ is a continuous separating map in the sense of 21]. By [21 Proposition 2], the space $X$ is metrizable.

$(3) \Rightarrow(1)$. This follows from Theorem 4.6

\section{REMARKS AND OPEN QUESTIONS}

The set $\mathbf{E}(X)$ of all regular extension operators of partial (pseudo)metrics on $X$ is a topological invariant of $X$. One can consider this set in different topologies, say, in the uniform convergence topology, compact-open topology, and pointwise convergence topology.

Question 7.1. If $\mathbf{E}\left(X_{1}\right)$ and $\mathbf{E}\left(X_{2}\right)$ are (topologically) isomorphic with respect to the natural convex structure in them (and one of the topologies mentioned above), are the compact metrizable spaces $X_{1}$ and $X_{2}$ homeomorphic? 
The following question is motivated by the results of [3].

Question 7.2. Are there linear extension operators $u: \mathcal{P M} \rightarrow \mathcal{P} \mathcal{M}(X)$ of norm 1 that are also continuous in the pointwise convergence topology on every $\mathcal{P} \mathcal{M}(A)$, where $A \in \exp X$ ?

Note that the implication $(2) \Rightarrow(3)$ from Theorem 6.1 can be generalized on the class of paracompact $p$-spaces (a topological space is a paracompact $p$-space if it admits a perfect map onto a metric space). The proof remains the same.

\section{REFERENCES}

[1] T. Banakh, On linear regular operators extending (pseudo)metrics, preprint.

[2] T. Banakh, $\mathrm{AE}(0)$-spaces and regular operators extending (averaging) pseudometrics, Bull. Polish Acad. Sci. Math. 42 (1994), no. 3, 197-206.

[3] T. Banakh and C. Bessaga, On linear operators extending (pseudo)metrics, Bull. Polish Acad. Sci. Math. 48 (2000), no. 1, 35-49. MR 2001e:54023

[4] C. Bessaga, On linear operators and functors extending pseudometrics, Fund. Math. 142 (1993), no. 2, 101-122. MR 94h:54033

[5] C. Bessaga, Functional analytic aspects of geometry. Linear extending of metrics and related problems, Progress in Functional Analysis (Peñíscola, 1990), 247-257, North-Holland Math. Stud., 170, North-Holland, Amsterdam, 1992. MR 93a:54015

[6] R. H. Bing, Extending a metric, Duke Math. J. 14 (1947), 511-519. MR 9:521c

[7] J. Dugundji, An extension of Tietze's theorem, Pacific J. Math. 1 (1951). 353-367. MR 13:373c

[8] N. Dunford and J. T. Schwartz, Linear operators. Part I. General theory, with the assistance of William G. Bade and Robert G. Bartle. Reprint of the 1958 original. Wiley Classics Library. A Wiley-Interscience Publication. John Wiley \& Sons, Inc., New York, 1988. xiv+858 pp. MR 22:8302

[9] V. V. Filippov, Topological structure of solution spaces of ordinary differential equations (in Russian), Uspekhi Mat. Nauk 48 (1993), 103-154; English transl., Russian Math. Surveys 48 (1993), no. 1, 101-154. MR 94f:34008

[10] A. Fryszkowski, Continuous selections for a class of non-convex multi-valued maps, Studia Math. 76 (1983), 163-174. MR 85j:54022

[11] M. Gromov, Metric structures for Riemannian and non-Riemannian spaces, Based on the 1981 French original [ MR 85e:53051]. With appendices by M. Katz, P. Pansu and S. Semmes. Translated from the French by Sean Michael Bates. Progress in Mathematics 152, Birkhäuser Boston, Inc., Boston, MA, 1999. xx+585 pp. MR 2000d:53065

[12] F. Hausdorff, Erweiterung einer Homöomorphie, Fund. Math. 16 (1930), 353-360.

[13] F. Hiai and H. Umegaki, Integrals, conditional expectations and martingales of multivalued functions, J. Multivariate Anal. 7 (1977), 149-182. MR 58:22463

[14] K. Kuratowski, Sur l'espace des fonctions partielles, Ann. Mat. Pura Appl. 40 (1955), 61-67. MR 17:650b

[15] K. Kuratowski, Sur une méthode de métrisation complète de certains espaces d'ensembles compacts, Fund. Math. 43 (1956), 114-138. MR 18:58a

[16] R. T. Rockafellar, Integrals which are convex functionals, Pacific J. Math. 24 (1968), 525-539. MR 38:4984

[17] H.-P. Künzi and L. B. Shapiro, On simultaneous extension of continuous partial functions, Proc. Amer. Math. Soc. 125 (1997), 1853-1859. MR 98g:54015

[18] Nguyen Van Khue and Nguyen To Nhu, Two extensors of metrics, Bull. Acad. Polon. Sci. Sér. Sci. Math. 29 (1981), no. 5-6, 285-291. MR 83g:54040

[19] J. Luukkainen, Extension of locally uniformly equivalent metrics, Colloq. Math. 46 (1982), no. 2, 205-207. MR 84a:54054

[20] O. Pikhurko, Extending metrics in compact pairs, Mat. Stud. 3 (1994), 103-106, 122.

[21] E. N. Stepanova, Continuation of continuous functions and the metrizability of paracompact p-spaces (Russian) Mat. Zametki 53 (1993), no. 3, 92-101; translation in Math. Notes 53 (1993), no. 3-4, 308-314. MR 94k:54031 
[22] H. Toruńczyk, A short proof of Hausdorff's theorem on extending metrics, Fund. Math. $\mathbf{7 7}$ (1972), no. 2, 191-193. MR 47:9559

[23] M. Zarichnyi, Regular linear operators extending metrics: a short proof, Bull. Polish Acad. Sci. Math. 44 (1996), no. 3, 267-269. MR 97m:54050

Department of Mathematics and Statistics, University of Saskatchewan, Room 142 McLean Hall, 106 Wiggins Road, Saskatoon, Saskatchewan, Canada S7N 5E6

E-mail address: tymchat@math.usask.ca

Department of Mechanics and Mathematics, Lviv National University, UniversytetSKA 1, 79000 LVIV, UKRAINE

E-mail address: topos@franko.lviv.ua

E-mail address: mzar@litech.lviv.ua 